\title{
DESARTERIALIZAÇÃO TRANSANAL GUIADA POR DOPPLER ASSOCIADA AO REPARO ANORRETAL NA DOENÇA HEMORROIDÁRIA: A TÉCNICA DO THD
}

\author{
Transanal desarterialization guided by Doppler associated to anorectal repair in hemorrhoids: THD technic \\ Carlos Walter SOBRADO-JUNIOR, José Américo Bacchi HORA
}

Trabalho realizado na Disciplina de Coloproctologia da Faculdade de Medicina da Universidade de São Paulo, SP, Brasil.

DESCRITORES - Cirurgia. Hemorroidas. Técnica.
RESUMO - Introdução - O tratamento cirúrgico para hemorroidas deve ter indicação individual e baseia-se no sintoma predominante (sangramento ou prolapso), na gravidade da doença e na presença ou ausência de componente externo (plicoma). Cabe ao cirurgião conhecer as mais variadas técnicas para que possa encontrar o tratamento mais adequado caso a caso. Técnica - O procedimento THD consiste na ligadura alta seletiva e guiada por Doppler de até seis ramos arteriais submucosos que irrigam as hemorroidas, levando à sua desarterialização, associada à correção do prolapso (reparo anorretal ou lifting). Utiliza equipamento e kit especiais. Conclusão - A técnica do THD tem mostrado bons resultados iniciais. Por ser técnica cirúrgica em que se respeita a anatomia, ela age diretamente sobre a fisiopatologia da afecção e corrige suas principais consequências, ela parece bastante promissora. Sua aplicação inicial pode ser nos pacientes com doença hemorroidária de II grau, que tenham indicação de tratamento cirúrgico, e de III e IV graus, nestes últimos, podendo ser associada à ressecção de plicomas.

\section{Correspondência:}

Carlos Walter Sobrado Junior,

e-mail: cwsobrado@hotmail.com

Fonte de financiamento: não há

Conflito de interesses: não há

Recebido para publicação: 25/06/2012

Aceito para publicação: 13/08/2012

HEADINGS - Surgery. Hemorrhoids. Technique
ABSTRACT - Introduction - Surgical treatment for hemorrhoids should be indicated individually and is based on the predominant symptom (bleeding or prolapse), severity of disease and the presence or absence of external component (plicoma). Surgeons must choose among varied techniques the one suitable for each case. Technic - The THD procedure consists of Doppler guided high ligation, selective to up six submucosal arterial branches that supply the hemorrhoids, leading to its desarterialization associated with prolapse repair (anorectal repair or lifting). It uses special equipment and kit. Conclusion - THD technique has shown good initial results. Because surgical technique respects the anatomy, it acts directly on the pathophysiology of the disease and corrects its principal consequences; it looks quite promising. Its initial application may be in patients with hemorrhoids grade II, which have surgical indication, grades III and IV, the latter being associated with resection of plicomas.

\section{INTRODUÇÃO}

busca pelo tratamento cirúrgico ideal da doença hemorroidária
remonta a tempos históricos, atualmente sendo necessário em
apenas $10-20 \%$ dos pacientes sintomáticos ${ }^{3}$. No último século, muitas técnicas foram desenvolvidas, mas poucas se firmaram como as propostas por Milligan-Morgan ${ }^{21}$, Parks ${ }^{24}$ e Ferguson ${ }^{8}$, que ainda podem ser consideradas o padrão-ouro no tratamento operatório desta afecção. Com o intuito de simplificar o processo terapêutico, também desenvolveramse modalidades de tratamento ambulatorial, como a escleroterapia ${ }^{12}$, a ligadura elástica ${ }^{2}$, a crioterapia ${ }^{19} \mathrm{e}$ a fotocoagulação ${ }^{23}$. Chegando à era da cirurgia minimamente invasiva, foram criados os métodos de anopexia mecânica ${ }^{20}$ e, mais recentemente, a desarterialização hemorroidária guiada por Doppler, ou THD (Transanal hemorrhoidal dearterialization) objeto deste 
texto. A terapêutica a ser instituída individualmente se baseia no sintoma predominante (sangramento ou prolapso), na gravidade da doença e na presença ou ausência de componente externo (plicoma). Cabe ao proctologista experiente conhecer as mais variadas técnicas para que possa encontrar o tratamento mais adequado caso a caso.

\section{Bases anatomofisiológicas da técnica}

O comprimento do canal anal varia de 2,5 a $4 \mathrm{~cm}$ acima da borda anal. Inferiormente ele se limita com a pele perianal, área altamente inervada e sensível. Tem seu revestimento epitelial dividido pela linha denteada ou linha pectínea em duas áreas: a cranial, menos sensível à dor, com inervação visceral, e a caudal, mais sensível. O suprimento sanguíneo para o reto e o canal anal é fornecido por três artérias: a artéria retal superior, ramo da artéria mesentérica inferior; artéria retal média, ramo da artéria ilíaca interna; e artéria retal inferior, ramo da artéria pudenda interna. O plexo hemorroidário interno, também chamado de corpo cavernoso do reto, aparece histologicamente como uma rede cavernosa venosa, com shunts arteriovenosos, cercados por tecido conjuntivo, dentro ou abaixo da mucosa retal. Este plexo recebe seu suprimento sanguíneo exclusivamente dos ramos terminais da artéria retal superior.

Segundo Aigner ${ }^{1}$, em indivíduos saudáveis, o diâmetro médio dos ramos terminais da artéria retal superior é 0,92 mm, com fluxo médio de $11,9 \mathrm{~cm} / \mathrm{sec}$ e em pacientes portadores da doença hemorroidária, tanto o diâmetro, quanto o fluxo aumentam, sendo o diâmetro médio de $1,87 \mathrm{~mm}$, com fluxo médio de $33,9 \mathrm{~cm} / \mathrm{sec}$. Estudos recentes ${ }^{28}$, com ultrassonografia Doppler endoanal $\left(360^{\circ}\right.$ - rotating ultrasonographic endoprobe) demonstraram que na circunferência da parede do reto distal, até $6 \mathrm{~cm}$ acima da margem anal, são geralmente identificados seis ramos arteriais localizados nas posições de 1, 3, 5, 7, 9 e 11 horas, em portadores ou não de doença hemorroidária. Neste mesmo estudo demonstraram que, nos pacientes portadores de doença hemorroidária, existe aumento da velocidade do fluxo sanguíneo arterial e diminuição na resistência venosa.

$\mathrm{Na}$ teoria do hiperfluxo arterial, o sangue arterial vai para os plexos hemorroidários com maior velocidade levando à congestão e lesão venosa, o que determina estase, edema e prolapso dos mamilos hemorroidários. A hipertensão nos coxins hemorroidários pelo hiperfluxo, contribui também para a degeneração do tecido conectivo. O aumento da sobrecarga durante a defecação piora ainda mais a drenagem venosa e a hipertensão nos coxins. Todo este estresse no tecido conectivo leva, com o tempo, à sua degeneração e ao aparecimento dos sintomas de prolapso, sangramento, desconforto, ardência, mucorréia, dor e prurido.

\section{Evolução histórica}

O tratamento por desarterialização hemorroidária guiada por Doppler para a doença hemorroidária - HAL (apenas desarterialização) foi inicialmente descrito no Japão em 1995, por Morinaga et al.22, que relataram bons resultados nos 116 pacientes avaliados, quanto à dor, sangramento e prolapso, após um mês de acompanhamento. Um grupo norteamericano em 2001, após operações em 60 pacientes, concluiu que o procedimento já poderia ser opção à hemorroidectomia, na maioria dos $\operatorname{casos}^{32}$. Desde então, tanto a técnica de desarterialização $\mathrm{HAL}$, com a introdução posterior do reparo (lifting) anorretal, quanto os equipamentos vêm sendo aperfeiçoados. Dados colhidos de séries de casos cada vez mais longas e com maior seguimento têm sido publicadas, principalmente de serviços na Europa $^{6}$. Atualmente a técnica THD (desarterialização associada à hemorroidopexia) têm sido indicada para doença hemorroidária de $2^{\circ}$ grau refratária, de $3^{\circ}$ grau e casos selecionados de $4^{\circ}$ grau.

\section{TÉCNICA}

O procedimento consiste na ligadura alta seletiva e guiada por Doppler de até seis ramos arteriais submucosos que irrigam as hemorroidas, levando à sua desarterialização, associada à correção do prolapso (reparo anorretal ou lifting).

Foi desenvolvido um anuscópio especial acoplado, na parte lateral de sua ponta, a um sensor Doppler, o que permite escutar o som do pulso arterial na submucosa, localizando a artéria. Faz-se toque digital suave com gel anestésico ou lubrificante, em seguida introduz-se o anuscópio. Identificam-se, por meio do som emitido, os ramos arteriais com grande precisão e faz-se sua ligadura com fio absorvível 00 (acido poliglicólico) à aproximadamente $6 \mathrm{~cm}$ acima da linha pectínea, com um ponto realizado através de uma janela lateral no instrumento. Esta desarterialização inicial já reduz o ingurgitamento do tecido hemorroidário, facilitando sua redução, suspensão e fixação (Figura 1).

Posteriormente, realiza-se um chuleio contínuo simples com o mesmo fio em direção craniocaudal até local cerca de $1,0 \mathrm{~cm}$ acima da linha pectínea, que, depois de amarrado ao ponto inicial, levará à pexia da mucosa e do tecido hemorroidário prolapsados, que retornam à sua posição anatômica. Nesta fase, deve-se evitar pontos profundos que envolvam o esfíncter anal, pois dificultam o lifting do tecido prolapsado, e também pontos ao nível da linha pectínea, que aumentam a dor no pós-operatório. Este procedimento deve ser realizado nos seis ramos arteriais, localizados nas posições $1,3,5,7,9,11$ horas. Finalmente, revisa-se a hemostasia e faz-se um curativo com a introdução de um hemostático que faz parte do kit THD (Figuras 2 e 3 ). 


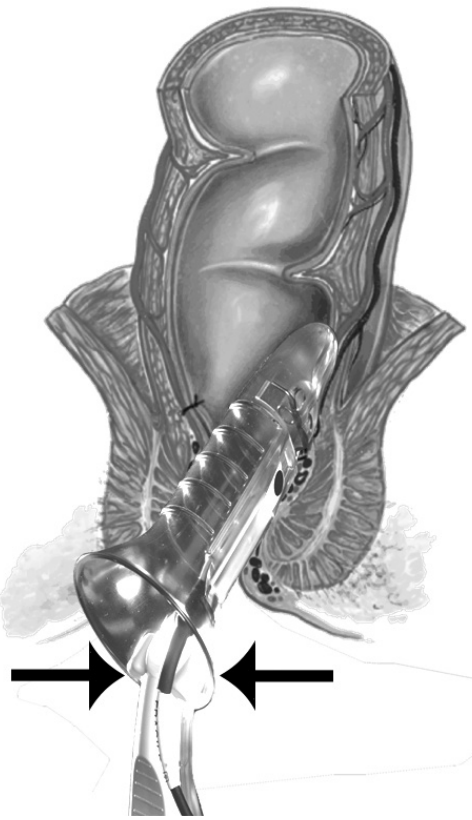

FIGURA 1 - Localização do pulso arterial com Doppler para realização do ponto inicial da ligadura. (Fonte: Carlo Ratto)

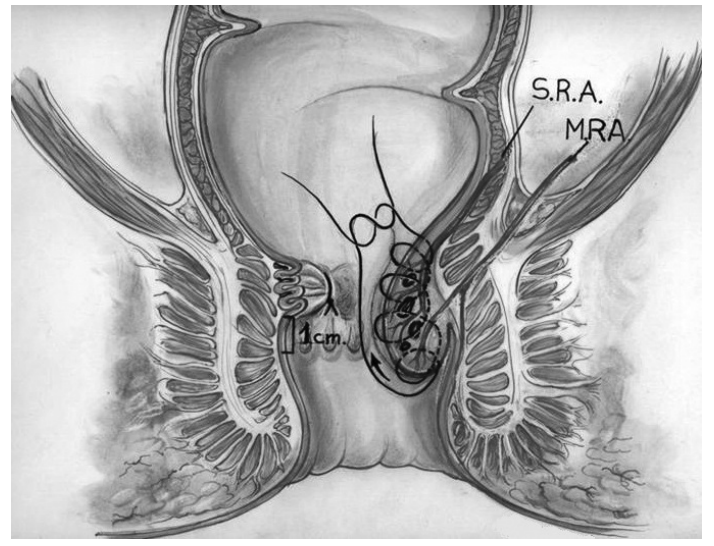

$\mathrm{SRA}=$ artéria retal superior. $\mathrm{MRA}=$ artéria retal média

FIGURA 2 - Representação final do chuleio para o reparo anorretal (lifting ou hemorroidopexia). (Fonte: Carlo Ratto)

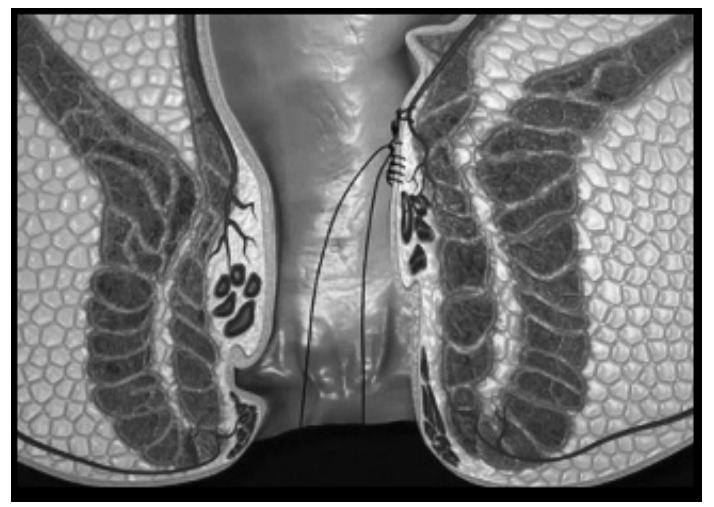

FIGURA 3 - Esquema do aspecto final do reparo anorretal (hemorroidopexia) e desarterialização hemorroidária. (Fonte: http://www.thdlab.com. br/tratamentos/1/curar-hemorroidas.html)
Assim, soma-se à interrupção do fluxo arterial, a pexia mucosa e hemorroidária, direcionando o tratamento à resolução dos dois principais sintomas da doença hemorroidária: o sangramento e o prolapso. Pelo fato de não haver ressecção tecidual e por todo o procedimento se dar acima da linha pectínea, esperase redução da dor pós-operatória, em relação aos procedimentos de hemorroidectomia. Antibióticos utilizam-se de rotina como profilaxia (Figuras 4 e 5).

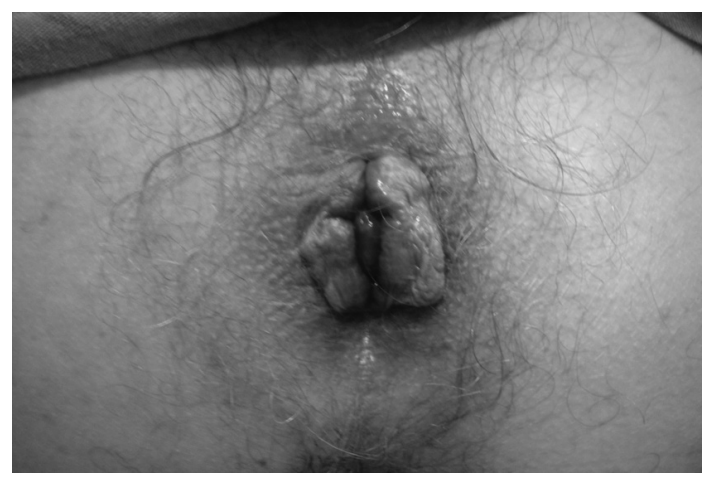

FIGURA 4 - Aspecto pré-operatório

Fibras comerciais (plantago ovata, psillium,

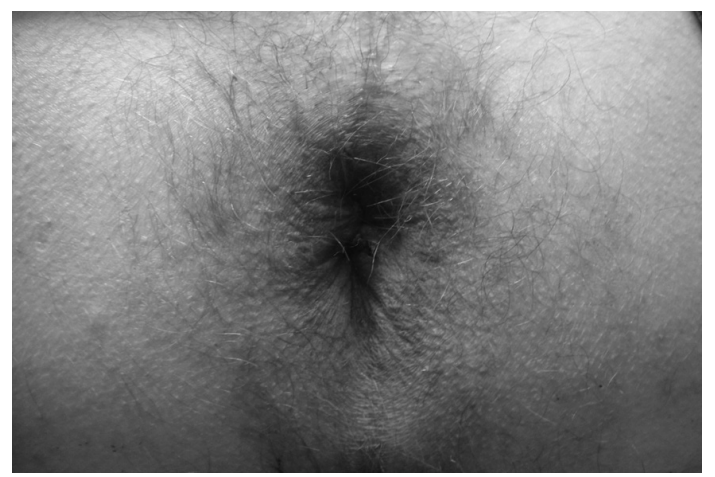

FIGURA 5 - Aspecto pós-operatório do THD

metilcelulose) são prescritas de rotina, assim como analgésicos (dipirona) e anti-inflamatórios (cetoprofeno). Todos os pacientes são examinados semanalmente por quatro semanas, quando são questionados com relação à dor, frequência evacuatória, aspecto das fezes, sangramento, satisfação com o procedimento e retorno ao trabalho.

\section{DISCUSSÃO}

Em 2009, em revisão de 1996 casos publicados na literatura, foi concluído que o tratamento é seguro para as hemorroidas graus II e III, e que são necessários mais estudos acerca de sua eficácia nos casos mais avançados e em longo prazo . Já em 2011, um grupo francês, em estudo não controlado com 100 pacientes com hemorroidas grau IV, obteve bons resultados com acompanhamento médio de 34 meses. No 
mesmo ano, Ratto et al. ${ }^{29}$ publicaram os resultados do procedimento em 35 pacientes consecutivos com doença hemorroidária mais avançada, obtendo dados semelhantes, o que leva a crer que a técnica tem potencial de emprego para a maioria dos casos com indicação cirúrgica $28,28,30,33$. Desde então, experiências iniciais realizadas em vários países $5,13,25,27,34$ já foram publicadas, com bons resultados, seja para o procedimento isolado ou associado à outras técnicas. Em nosso meio vem ganhando adeptos nos últimos anos, já tendo sido realizadas apresentações em congressos e publicações acerca desta experiência inicial ${ }^{16,31}$, que demonstram bons resultados e baixo índice de complicações em dois anos de acompanhamento.

Foram encontradas seis publicações de estudos comparativos utilizando a desarterialização hemorroidária. Quatro deles comparavam-na à anopexia mecânica, mostrando resultados semelhantes em médio prazo, com um estudo observando menor incidência de complicações precoces e mais rápido retorno ao trabalho para os grupos da desarterialização ${ }^{10}$, e dois, discreta diminuição da dor no pós-operatório precoce $e^{9,17}$. Um grupo chinês foi francamente favorável, quanto às complicações precoces e à dor no pós-operatório, ao grupo de desarterialização ${ }^{26}$. Em trabalho comparativo com a hemorroidectomia fechada e com esta técnica, mostrou-se menos dor nesta última e recuperação mais precoce, sendo semelhantes os resultados em um ano ${ }^{4}$. Estudo indiano não encontrou diferenças entre a desarterialização guiada por Doppler e a simples ligadura cirúrgica das hemorroidas em até um ano de seguimento ${ }^{14}$. Já estudo egípcio, demonstrou que a hemorroidectomia é superior ao PPH e à desarterialização nos casos avançados em que há plicomas anais, e semelhante nos demais ${ }^{15}$.

\section{CONCLUSÕES}

A técnica do THD tem mostrado bons resultados iniciais. Por ser técnica cirúrgica em que se respeita a anatomia, se age diretamente sobre a fisiopatologia da afecção e se corrige suas principais consequências, ela parece bastante promissora. Sua aplicação inicial pode ser nos pacientes com doença hemorroidária de II grau, que tenham indicação de tratamento cirúrgico, e de III e IV graus, nestes últimos, podendo ser associada à ressecção de plicomas.

\section{REFERÊNCIAS}

1. Aigner F, Bodner G, Conrad F, Mbaka G, Kreczy A, Fritsch H. The superior rectal artery and its branching pattern with regard to its clinical influence on ligation techniques for internal hemorrhoids. Am J Surg. 2004 Jan;187(1):102-8.

2. Barron J. Office ligation of internal hemorrhoids. Am J Surg. 1963 Apr;105:563-70.

3. Bleday R, Pena JP, Rothenberger DA, Goldberg SM, Buls JG. Symptomatic hemorrhoids: current incidence and complications of operative therapy. Dis Colon Rectum. 1992 May;35(5):477-81.
4. Bursics A, Morvay K, Kupcsulik P, Flautner L. Comparison of early and 1-year follow-up results of conventional hemorrhoidectomy and hemorrhoid artery ligation: a randomized study. Int J Colorectal Dis. 2004 Mar;19(2):176-80.

5. Charúa Guindic L, Fonseca Muñoz E, García Pérez NJ, Osorio Hernández RM, Navarrete Cruces T, Avendaño Espinosa O, Guerra Melgar LR. Hemorrhoidal desarterialization guided by Doppler. A surgical alternative in hemorrhoidal disease management. Rev Gastroenterol Mex. 2004 Apr-Jun;69(2):83-7.

6. Faucheron JL, Gangner Y. Doppler-guided hemorrhoidal artery ligation for the treatment of symptomatic hemorrhoids: early and three-year follow-up results in 100 consecutive patients. Dis Colon Rectum. 2008 Jun;51(6):945-9.

7. Faucheron JL, Poncet G, Voirin D, Badic B, Gangner Y. Dopplerguided hemorrhoidal artery ligation and rectoanal repair (HALRAR) for the treatment of grade IV hemorrhoids: long-term results in 100 consecutive patients. Dis Colon Rectum. 2011 Feb;54(2):226-31.

8. Ferguson JA, Mazier WP, Ganchrow MI, Friend WG. The closed technique of hemorrhoidectomy. Surgery. 1971 Sep;70(3):480-4.

9. Festen S, van Hoogstraten MJ, van Geloven AA, Gerhards MF. Treatment of grade III and IV haemorrhoidal disease with PPH or THD. A randomized trial on postoperative complications and short-term results. Int J Colorectal Dis. 2009 Dec;24(12):1401-5.

10. Giordano P, Nastro P, Davies A, Gravante G. Prospective evaluation of stapled haemorrhoidopexy versus transanal haemorrhoidal dearterialisation for stage II and III haemorrhoids: three-year outcomes. Tech Coloproctol. 2011 Mar;15(1):67-73.

11. Giordano P, Overton J, Madeddu F, Zaman S, Gravante G. Transanal hemorrhoidal dearterialization: a systematic review. Dis Colon Rectum. 2009 Sep;52(9):1665-71.

12. Graham-Stewart CW. Injection treatment of haemorrhoids. $\mathrm{Br}$ Med J. 1962 Jan 27;1(5273):213-6.

13. Greenberg R, Karin E, Avital S, Skornick Y, Werbin N. First 100 cases with Doppler-guided hemorrhoidal artery ligation. Dis Colon Rectum. 2006 Apr;49(4):485-9

14.Gupta PJ, Kalaskar S, Taori S, Heda PS. Doppler-guided hemorrhoidal artery ligation does not offer any advantage over suture ligation of grade 3 symptomatic hemorrhoids. Tech Coloproctol. 2011 Dec;15(4):439-44.

15. Hafagy W, El Nakeeb A, Fouda E, Omar W, Elhak NG, Farid M, Elshobaky M. Conventional haemorrhoidectomy, stapled haemorrhoidectomy, Doppler guided haemorrhoidectomy artery ligation; post-operative pain and anorectal manometric assessment. Hepatogastroenterology. 2009 Jul-Aug;56(93):1010-5.

16. Hora, JAB; Klajner, S; Kruglensky, D; Sobrado Jr, CW. Tratamento Cirúrgico da Doença Hemorroidária por Desarterialização Transanal Guiada por Doppler associada ao Reparo Anorretal (A Técnica do THD): Experiência Inicial. $1^{\circ}$ Congresso Paulista de Coloproctologia. São Paulo 8 e 9 de junho de 2012.

17.Infantino A, Altomare DF, Bottini C, Bonanno M, Mancini S. Prospective randomized multicentre study comparing stapler haemorrhoidopexy with Doppler-guided transanal haemorrhoid dearterialization for third-degree haemorrhoids. Colorectal Dis. 2012 Feb;14(2):205-211.

18. Jeong WJ, Cho SW, Noh KT, Chung SS. One Year Follow-up Result of Doppler-guided Hemorrhoidal Artery Ligation and Recto-Anal Repair in 97 Consecutive Patients. J Korean Soc Coloproctol. 2011 Dec;27(6):298-302.

19. Lewis MI. Diverse methods of managing hemorrhoids: cryohemorrhoidectomy. Dis Colon Rectum. 1973 MayJun;16(3):175-7. Neiger A (1989) Infrared-photo-coagulation for hemorrhoids treatment. Int Surg 74:142-143

20. Longo A. Treatment of haemorrhoids disease by reduction of mucosa and haemorrhoidal prolapse with a circular suturing device: a new procedure. Proceedings of the Sixth World Congress of Endoscopic Surgery. Monduzzi, 1998;777-84.

21. Milligan ETC, Morgan C, Naughton JLF, Office RR. Surgical anatomy of the anal canal and the operative treatment of haemorrhoids. Lancet 1937; II:1119-24 
22. Morinaga K, Hasuda K, Ikeda T. A novel therapy for internal hemorrhoids: ligation of the hemorrhoidal artery with a newly devised instrument (Moricorn) in conjunction with a Doppler flowmeter. Am J Gastroenterol. 1995 Apr;90(4):610-3.

23. Neiger A. Infrared-photo-coagulation for hemorrhoids treatment. Int Surg. 1989 Jul-Sep;74(3):142-3.

24. Parks AG. The surgical treatment of haemorrhoids. Br J Surg. 1956 Jan;43(180):337-51.

25. Pol RA, van der Zwet WC, Hoornenborg D, Makkinga B, Kaijser M, Eeftinck Schattenkerk M, Eddes EH. Results of 244 consecutive patients with hemorrhoids treated with Doppler-guided hemorrhoidal artery ligation. Dig Surg. 2010;27(4):279-84.

26. Qin PP, Huang B, Cai HJ, Ge Q Wang ZL. A prospective controlled comparative study of Doppler-guided hemorrhoidal artery ligation versus operative procedures for prolapse and hemorrhoids. Zhonghua Yi Xue Za Zhi. 2010 Nov 30;90(44):3131-4.

27. Ramírez JM, Aguilella V, Elía M, Gracia JA, Martínez M. Doppler-guided hemorrhoidal artery ligation in the management of symptomatic hemorrhoids. Rev Esp Enferm Dig. 2005 Feb;97(2):97-103.

28. Ratto C, Donisi L, Parello A, Litta F, Doglietto GB. Evaluation of transanal hemorrhoidal dearterialization as a minimally invasive therapeutic approach to hemorrhoids. Dis Colon Rectum. 2010 May;53(5):803-11.
29. Ratto C, Giordano P, Donisi L, Parello A, Litta F, Doglietto GB. Transanal haemorrhoidal dearterialization (THD) for selected fourth-degree haemorrhoids. Tech Coloproctol. 2011 Jun;15(2):191-7.

30. Scheyer M. Doppler-guided recto-anal repair: a new minimally invasive treatment of hemorrhoidal disease of all grades according to Scheyer and Arnold. Gastroenterol Clin Biol. 2008 Jun-Jul;32(6-7):664

31.Sobrado Jr CW, Hora JAB. Tratamento Cirúrgico da Doença Hemorroidária por Desarterialização Transanal Guiada por Doppler associada ao Reparo Anorretal: A Técnica do THD. Atualização em Cirurgia do Aparelho Digestivo e em Coloproctologia - São Paulo: Departamento de Gastroenterologia da Faculdade de Mediccina da USP, 2012. Jul 2012: 319-326.

32. Sohn N, Aronoff JS, Cohen FS, Weinstein MA. Transanal hemorrhoidal dearterialization is an alternative to operative hemorrhoidectomy. Am J Surg. 2001 Nov;182(5):515-9.

33. Testa A, Torino G, Gioia A. DG-RAR (Doppler-guided rectoanal repair): a new mini invasive technique in the treatment of prolapsed hemorrhoids (grade III-IV): preliminary report. Int Surg. 2010 Jul-Sep;95(3):265-9.

34. Toh EL, Ng KH, Eu KW. The fourth branch of the superior rectal artery and its significance in transanal haemorrhoidal dearterialisation. Tech Coloproctol. 2010 Dec;14(4):345-8. 